\title{
Méthode empirique d'évaluation du coefficient de réaction du sol vis-à-vis des ouvrages de soutènement souples
}

P. SCHMITT

SOLÉTANCHE

6, rue de Watford 92000 Nanterre
Cet article reprend les résultats de mesures effectuées sur une dizaine d'ouvrages de soutènement (parois moulées et rideaux de palplanches), qui avaient déjà fait l'objet d'une interprétation en 1984 dans le $n^{\circ} 28$ de la Revue Française de Géotechnique.

La synthèse effectuée cette fois-ci débouche sur une expression simple et générale du coefficient de réaction du sol, tenant compte de deux facteurs dont l'importance a été clairement mise en évidence par les mesures sur ouvrages réels, à savoir la non-linéarité du comportement du sol et l'inertie du soutènement.

\section{Estimating the coefficient of subgrade reaction for diaphragm wall and sheet pile wall design}

This article reviews site measurements from about ten diaphragm wall or sheet pile wall works, which had already been analysed in Revue Française de Géotechnique, $n^{\circ} 28,1984$. The present synthesis results in a simple and general expression of the coefficient of subgrade reaction, taking into account two essential factors according to site experiments, which are the non-linear soil behaviour and the inertia of the retaining structure. 


\section{Introduction}

En 1984, une étude expérimentale [8], exploitant les résultats obtenus à partir d'une dizaine de chantiers instrumentés, avait permis de dégager quelques conclusions concernant le « coefficient de réaction » utilisé pour le calcul des parois moulées et rideaux de palplanches.

Au cours des dix dernières années, ces conclusions ont permis, sans subir de démenti expérimental, d'étayer le choix des hypothèses de calcul d'un grand nombre de projets.

Mais, faute, probablement, d'avoir pu déboucher sur une formulation générale, cette approche empirique se voit souvent préférer l'application exclusive d'autres méthodes, par exemple celles proposées par Balay [2] ou Chadeisson [7], dont elle pourrait cependant constituer un complément intéressant en raison de son large support expérimental.

Il nous a donc semblé utile de rappeler les conclusions de cette étude, et d'en proposer une formulation suffisamment explicite pour être directement utilisable.

On rappellera, dans un premier temps, ce que l'on entend par "coefficient de réaction $)$ dans le cas des ouvrages de soutènement.

\section{2}

\section{Définition et méthodes d'évaluation du coefficient de réaction}

La méthode actuellement la plus répandue en France pour calculer les parois moulées et rideaux de palplanches est la méthode dite " élasto-plastique » (par opposition aux calculs à la rupture traditionnels), dans laquelle la phase élastique du comportement du sol est schématisée, en l'absence de chargements localisés, par la relation :

$$
\mathrm{p}=\mathrm{K}_{\mathrm{o}} \cdot \sigma_{\mathrm{v}}+1-\mathrm{k} \cdot \mathrm{y}
$$

dans laquelle p est la contrainte horizontale exercée par le sol sur l'ouvrage, $\mathrm{K}_{0}$ le coefficient de poussée des terres au repos, $\sigma$ la contrainte verticale existant dans le sol à la profondeur considérée, $\mathrm{k}$ le coefficient de réaction du sol, et y le déplacement horizontal du soutènement.

Le coefficient de réaction est donc le rapport, supposé constant pour une couche de sol donnée (bien que cela ne repose sur aucun fondement théorique), de la variation de contrainte horizontale au déplacement associé.

On entend ainsi trnir compte, à travers cet artifice de calcul, de l'enser ble des propriétés élastiques du sol et de l'ensemble les conditions aux limites du projet : on comprend $c$ nc que la détermination du coefficient de réaction, ' „i supposerait en toute rigueur des calculs prélimina: s plus élaborés que le calcul élastoplastique lui-mêr $\geqslant$, constitue un problème difficile qui divise encore le spécialistes.

Bien que d $\varsigma$. ordres de grandeur de $\mathrm{k}$ aient été indiqués dès 195. par Terzaghi [10], il est généralement admis que les valeurs réelles leur sont nettement supérieures, et les méthodes d'évaluation les plus fréquemment appliquées aujourd'hui sont :

- l'utilisation de la formule de Ménard et Bourdon [6] :

$$
\mathrm{k}=\mathrm{E}_{\mathrm{M}} /\left[\alpha, \mathrm{a} / 2+0,13 \cdot(9 \cdot \mathrm{a})^{\alpha}\right]
$$

dans laquelle $E_{M}$ est le module pressiométrique du sol, $\alpha$ le $\alpha$ coefficient de structure $n$, et a la hauteur sur laquelle le sol est sollicité en butée par l'ouvrage, que Ménard évalue forfaitairement aux $2 / 3$ de la fiche du rideau :

- l'utilisation de la formule de Marche [5] :

$$
\mathrm{k}=4,5 \cdot \mathrm{q}_{\mathrm{c}} / \mathrm{a}
$$

dans laquelle $q_{c}$ est la résistance de pointe au pénétromètre statique, et qui conduit, bien qu'établie à l'origine pour calculer, non pas les soutènements, mais les pieux sollicités horizontalement par le terrain, à des ordres de grandeur tout à fait comparables à ceux obtenus à partir de la formule (2) ;

- l'utilisation des ordres de grandeur proposés par Chadeisson, dont Monnet a récemment publié une formulation générale [7];

- la méthode pressiométrique établie par Balay [2], qui complète celle de Ménard par une méthode d'évaluation de a applicable aux étapes de terrassement intermédiaires, et par une formulation de $\mathrm{k}$ spécifique aux phases de mises en tension des tirants précontraints.

Compte tenu des divergences encore importantes entre les résultats déduits de ces différentes méthodes d'évaluation de $k$, d'une part, et compte tenu de la difficulté d'aborder de façon théorique l'évaluation d'un paramètre sans signification physique bien précise, d'autre part, Solétanche avait donc procédé, au début des années 80 , à la réalisation et à l'exploitation d'une série de mesures sur ouvrages réels, en intégrant également différents résultats ayant fait par ailleurs l'objet de communications.

\section{3 \\ Rappel des conclusions de l'étude de 1984 [8]}

La comparaison systématique entre résultats théoriques et expérimentaux obtenus sur neuf sites différents, et concernant des ouvrages allant du rideau de palplanches Larssen IIs à la paroi moulée d'1,33 m d'épaisseur, avait permis de dégager les conclusions suivantes :

1. La méthode élasto-plastique permet, malgré le caractère discutable de ses fondements théoriques, de rendre compte du comportement réel des ouvrages de façon satisfaisante (exemples des figures 1 et 2), sous réserve des conditions suivantes:

- choix approprié (voir ci-après) des paramètres de calcul, et notamment de la valeur du coefficient de réaction :

- limitation de l'usage de la méthode à son domaine d'application, à savoir le calcul des sollicitations dans les cas où celles-ci ne sont pas notablement affectées par les déformations d'ensemble du massif de sol (cas, 


\section{Tube inclinométrique $\mathrm{N}^{\circ} 4$}

Etat initial : 20-3-81

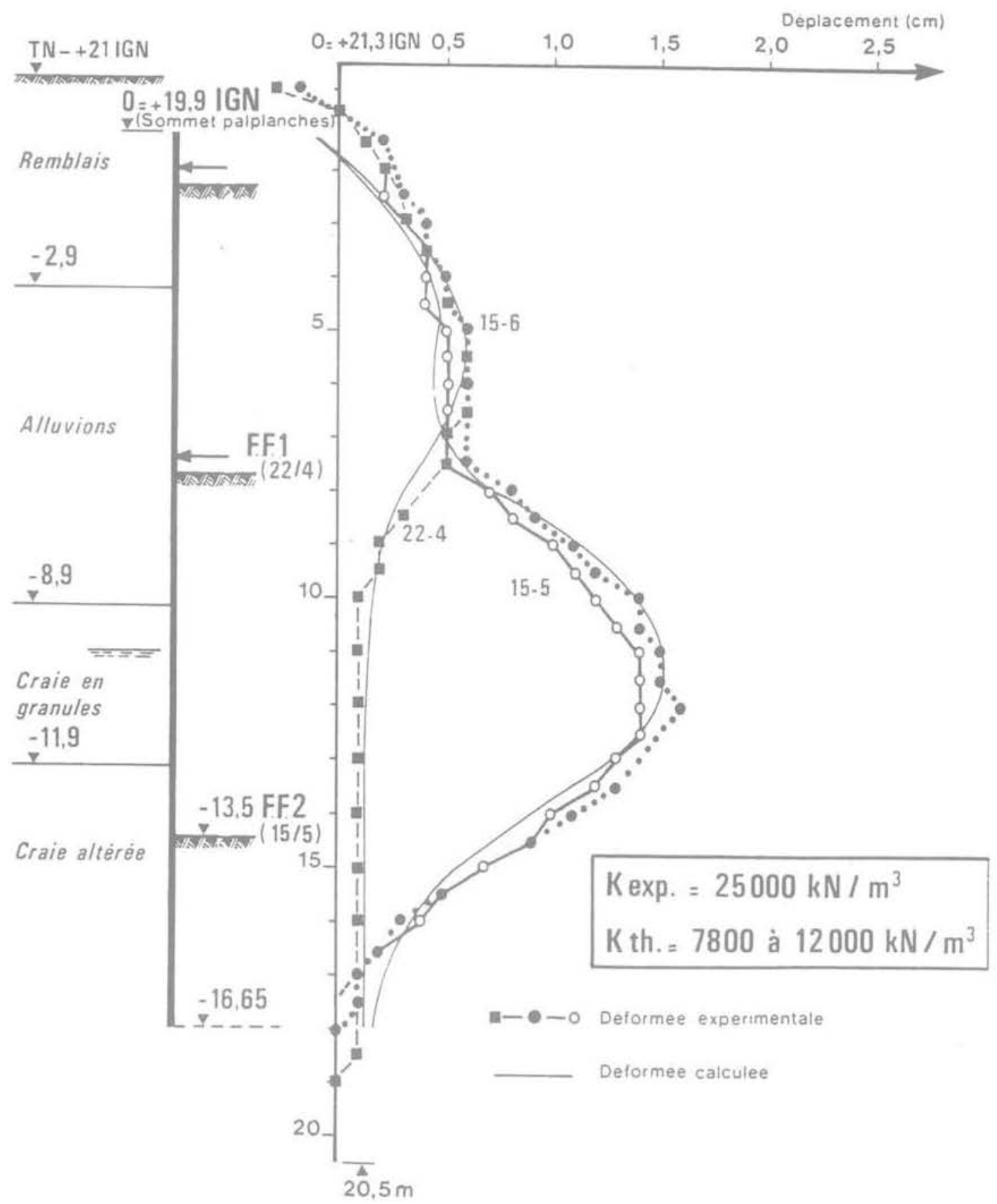

FGG.1 Lille-Déformée du rideau de palplanches. Lille - Deflection of the sheet pile wall

notamment, des grandes excavations stabilisées par des nappes d'ancrages multiples [4]).

2. Le coefficient de réaction expérimental (obtenu par ajustement entre le calcul et les résultats des mesures] est deux à quatre fois supérieur au coefficient théorique obtenu à partir de l'essai pressiométrique interprété suivant la formule de Ménard et Bourdon tant que le taux de mobilisation de la butée n'excède pas $50 \%$, ce taux de mobilisation étant conventionnellement pris égal, dans le cas d'un terrain sans cohésion, à la valeur moyenne de $\mathrm{k} \cdot \mathrm{y} /\left(\left(\mathrm{K}_{\mathrm{p}}-\mathrm{K}_{\mathrm{o}}\right) \cdot \sigma_{\mathrm{v}}\right)$, où $\mathrm{K}_{\mathrm{p}}$ est le coefficient de butée calculé dans l'hypothèse d'une inclinaison des contraintes sur la normale à l'écran égale à l'angle de frottement interne du sol.

Cette constatation peut être expliquée par la nonlinéarité du comportement du sol, et conduit à propo- 


\section{MESURES INCLINOMETRIOUES}

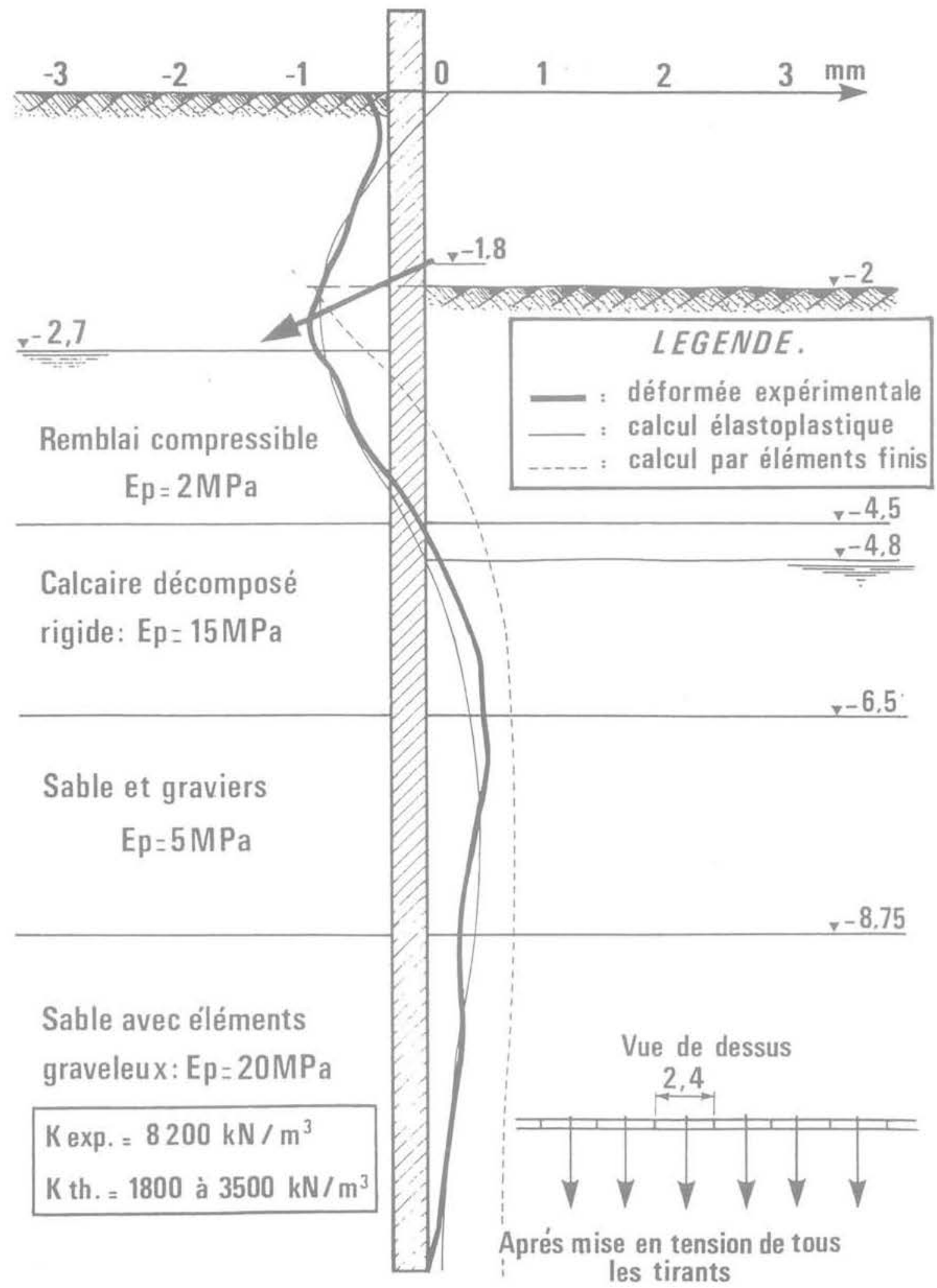

ser la relation pression-déplacement de la figure 3 , qui revient en pratique, compte tenu des coefficients de sécurité usuels, à retenir un ordre de grandeur « réaliste » du coefficient $\mathrm{k}$ égal à trois fois celui obtenu à partir de la formule de Ménard et Bourdon.
3. La valeur de k dépend directement de celle du paramètre dimensionnel a, hauteur sur laquelle le soutènement sollicite le terrain en butée, elle-même fonction de la rigidité relative du soutènement par rapport au sol. 


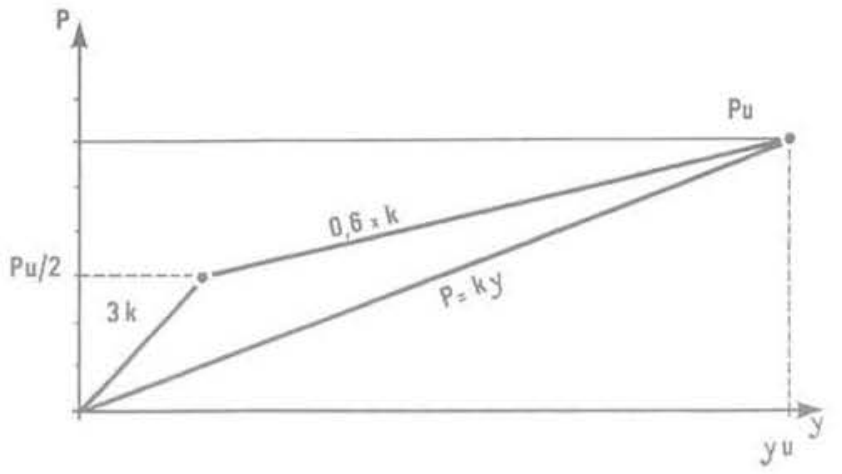

n..3 Comportement expérimental de l'interface sol-structure.

Experimental soil-structure interaction.

Dans l'étude expérimentale, la valeur théorique de $\mathrm{k}$ était calculée à partir de la valeur expérimentale de a (égale à la hauteur sur laquelle le déplacement réel du soutènement est " significatif », soit, en l'occurrence, supérieur à $20 \%$ du déplacement maximal).

Mais, lors de l'établissement d'un projet, la déformée réelle du soutènement n'est évidemment pas connue, et la valeur de a ne peut donc être qu'estimée de façon empirique, voire arbitraire ; il en résulte une indétermination importante dans l'évaluation de $\mathrm{k}$, qui contribue largement aux divergences souvent excessives observées entre les résultats des différentes méthodes de prévision.

La suite de cet article sera donc exclusivement consacrée au problème de l'évaluation de a.

\section{Observations expérimentales de " a "}

La figure 1 met en évidence, dans le cas d'un ouvrage de soutènement souple (palplanches Larssen IVs), des valeurs de a de l'ordre de $2 \mathrm{~m}$ (variables de $1,5 \mathrm{~m}$ à 2,5 $\mathrm{m}$ d'une phase de terrassement à l'autre, donc suivant le type de terrain et le mode de sollicitation).

On peut en tirer les conclusions suivantes:

1. Si la proposition de Ménard [6], qui consiste à retenir forfaitairement pour a une valeur égale aux $2 / 3$ de la fiche, s'avère parfaitement justifiée dans l'étape finale des terrassements, la généralisation de cette règle à l'étape intermédiaire de pose du $2^{\mathrm{C}}$ lit de butons conduirait à une valeur de a égale à $7 \mathrm{~m}$, soit près de 5 fois trop forte, donc à une valeur de $\mathrm{k}$ environ 5 fois trop faible, et même, globalement, 15 fois trop faible compte tenu de la non-linéarité du sol.

2. La faible valeur de a au cours des étapes de terrassement intermédiaires est vraisemblablement due à la faible rigidité du rideau par rapport au sol : il semblerait donc bien que la rigidité soit le paramètre principal $(\mathrm{a}=1,5 \mathrm{~m}$ au lieu de $7 \mathrm{~m})$, le mode de sollicitation, fonction des conditions de butonnage et de la hauteur terrassée, apparaissant comme un paramètre secondaire (a variable de $1,5 \mathrm{~m}$ à 2,5 $\mathrm{m}$ d'une étape de terrassement à l'autre).
L'importance de la rigidité de l'ouvrage est encore mise en évidence par la comparaison des figures 2 et 4 , qui correspondent à des mises en tension de tirants à faible profondeur (1,8 à 2,5 m) dans des terrains compressibles (module pressiométrique, $\mathrm{E}_{\mathrm{M}^{\prime}}$ variable de 2 à $3,5 \mathrm{MPa}$ ),

La différence essentielle entre les deux projets est l'épaisseur du soutènement, le quai de Deauville étant une paroi préfabriquée de $0,35 \mathrm{~m}$ et celui de Rouen une paroi moulée de $1,33 \mathrm{~m}$.

On constate, dans le premier cas, une déformation " de type souple ), localisée sur un peu plus de $3 \mathrm{~m}$ de hauteur, et dans le second cas une déformation « de type rigide $n$, significative sur plus de $10 \mathrm{~m}$ de hauteur.

Corrélativement, la valeur expérimentale du coefficient de réaction est plus de trois fois plus faible à Rouen quà Deauville, bien que le terrain y soit moins compressible : la rigidité relative du soutènement apparait donc ici comme un paramètre plus important que la compressibilité intrinsèque du sol.

Compte tenu de ces observations déjà anciennes, la valeur de a est le plus souvent choisie, au stade de la mise au point du projet, dans l'intervalle ( $2 \mathrm{~m}, 10 \mathrm{~m})$, en fonction de la rigidité du soutènement (avec bien entendu une borne supérieure égale aux $2 / 3$ de la fiche).

Mais cet intervalle relativement large laisse une place importante à l'appréciation subjective, et le besoin d'une méthode systématique d'évaluation de a se fait toujours sentir.

\section{5}

\section{Méthode d'évaluation de " a "}

Ces observations expérimentales conduisent à envisager l'hypothèse d'une proportionnalité de a à la longueur élastique du soutènement, ce qui revient à écrire, comme Westergaard l'avait déjà fait en 1926 dans le cas des radiers [11]:

$$
\mathrm{a}-(\mathrm{EI} / \mathrm{k})^{0.25}
$$

où EI est le produit d'inertie d'une unité de largeur du soutènement.

La théorie du « bulbe de pressions ») de Terzaghi [10] conduit, par ailleurs, à la conclusion que le produit $\mathrm{k}$. a, et non pas k lui-même, est une caractéristique intrinsèque du comportement élastique du sol (du moins pour des conditions aux limites données), ce qui permet d'écrire, pour une valeur donnée du coefficient de Poisson :

$$
k-E_{s} / a
$$

où $E_{\mathrm{s}}$ désigne le module d'Young du sol, ou toute grandeur qui lui est proportionnelle (module œedométrique, rapport $\mathrm{E}_{\mathrm{M}} / \alpha$ du module pressiométrique au « coefficient de structure $)$, résistance de pointe au pénétromètre statique, etc.).

La relation (5) traduit d'ailleurs simplement le fait qu'en élasticité linéaire, le déplacement en un point du milieu sollicité est proportionnel à la force qui lui est appliquée.

On déduit des relations (4) et (5) que a peut être directement exprimé en fonction du rapport $\mathrm{EL}_{\mathrm{E}} \mathrm{E}_{6}$ sous la forme :

$$
a \sim\left(E I / E_{S}\right)^{0.33}
$$

Cette formulation très simple pourrait par exemple s'appliquer à la détermination de $\mathrm{k}$ à partir de l'essai 

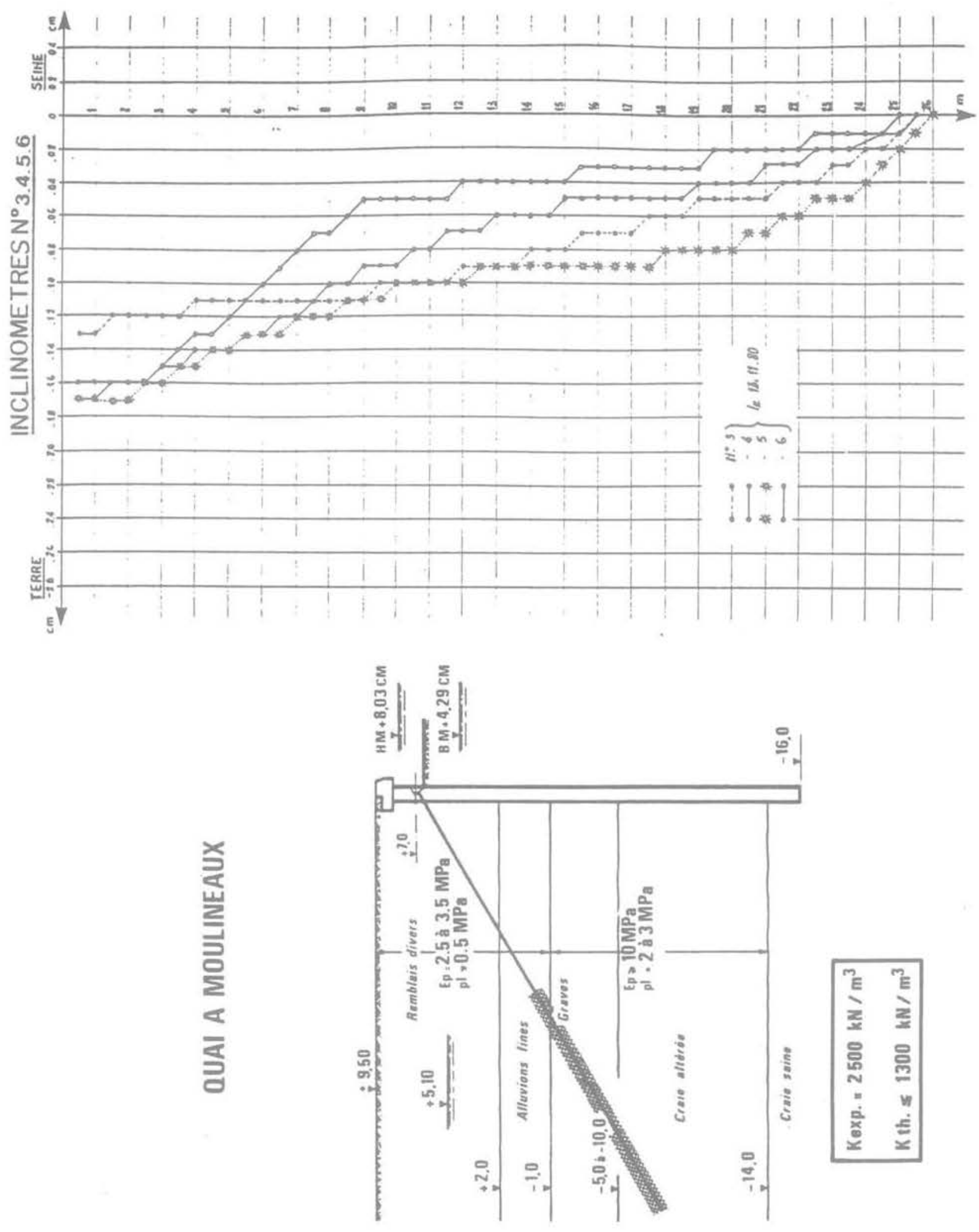

FG.4 Rouen-Déformée de la paroi moulée. Rouen - Deflection of the diaphragm wall.

pénétrométrique, puisque la formule de Marche (3), est bien du type de la formule (5).

Par contre, la formule de Ménard et Bourdon (2), est d'une forme plus complexe, et se prête donc moins bien à l'application de la formule (6).
Les figures 5 et 6 montrent toutefois qu'il est licite, pour les valeurs de a comprises dans l'intervalle $(2 \mathrm{~m}$, $10 \mathrm{~m}$ ), de lui substituer la formule simplifiée :

$$
k=1,2 \cdot E_{M} /(\alpha \cdot a)
$$




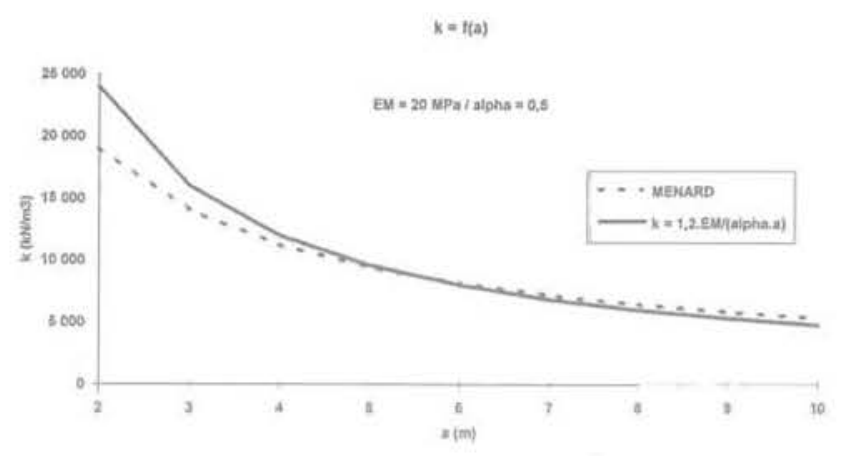

HIG.5 Expressions théoriques de $\mathrm{k}$ en fonction de a $(\alpha=0,5)$.

Theoretical relations between a and $k(\alpha=0,5)$.

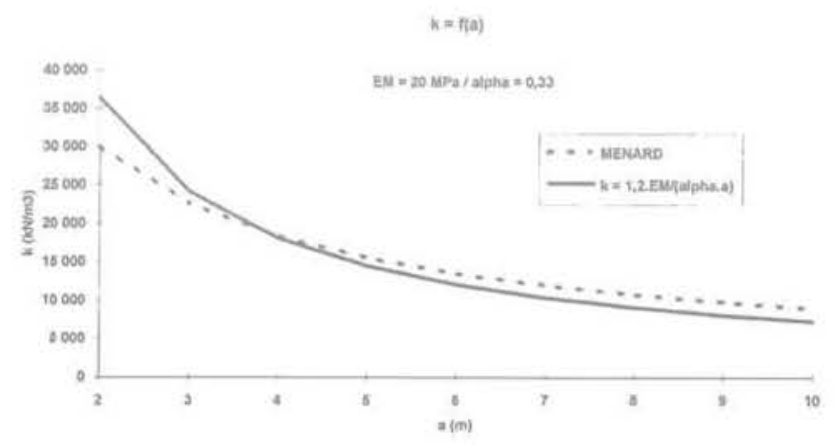

FG. 6 Expressions théoriques de $\mathrm{k}$ en fonction de a $(\alpha=0,33)$.

Theoretical relations between $\mathrm{a}$ and $\mathrm{k}(\alpha=0,33)$.

On peut dès lors, compte tenu de (5) et (6), envisager de mettre a sous la forme :

$$
\mathrm{a} \sim\left(\mathrm{EI} \cdot \alpha / \mathrm{E}_{\mathrm{M}}\right)^{0.33}
$$

On remarquera que les formules (7) et (8), a priori générales, sont du même type que celles proposées par Balay [2] et Gigan [3] pour la mise en tension des tirants précontraints.

Il n'en demeure pas moins qu'une validation de la formule (8) à partir de mesures sur ouvrages réels est indispensable.

C'est la raison pour laquelle on a reporté, sur la figure 7 , les valeurs expérimentales de a mesurées lors de l'étude de 1984, en fonction des valeurs correspondantes de $\mathrm{EI}, \alpha / \mathrm{E}_{\mathrm{M}}$.

En dépit d'une dispersion relativement importante, probablement imputable à l'imprécision des données expérimentales et à l'effet du mode de sollicitation, on met ainsi en évidene une relation nettement croissante, ce qui confirme la sensibilité du paramètre a à la rigidité du soutènement.

Toutefois, l'approximation linéaire de l'ensemble des résultats expérimentaux conduit à une droite de pente égale à 0,28 , ce qui, compte tenu des coordonnées bi-logarithmiques, suggère une relation intermédiaire entre une racine quatrième et une racine cubique.

Cette divergence avec la théorie et l'analyse dimensionnelle pourrait être la conséquence de l'hypothèse

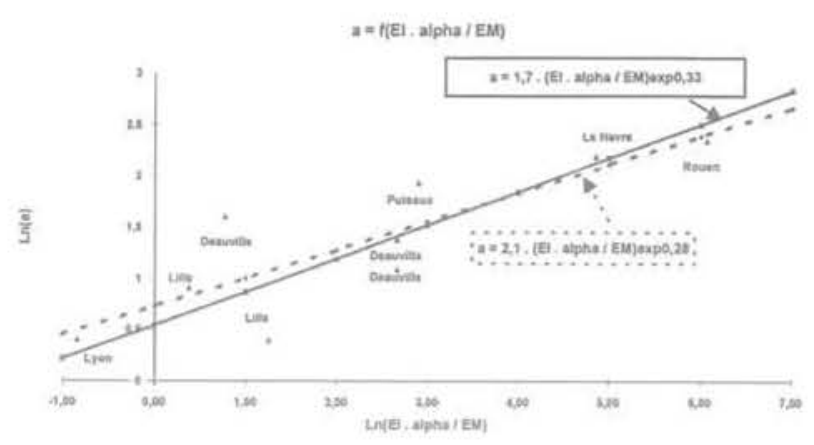

FG.7 Relation expérimentale entre a et EI. $\alpha / \mathrm{E}_{\mathrm{N}}$ Experimental relation between a and El $\alpha / \mathrm{E}_{\mathrm{M}^{*}}$

simplificatrice d'un coefficient de réaction constant avec la profondeur, implicitement admise dans l'expression de la longueur élastique du soutènement, et manifestement fausse dans le cas de multicouches très contrastés dans la zone de butée (cas où l'évaluation du coefficient de réaction s'avère d'ailleurs particulièrement délicate).

Après élimination des deux résultats expérimentaux qui se trouvent manifestement dans ce cas (étape finale du dragage à Deauville et mise en tension des tirants à Rouen), la pente de la droite de régression devient égale à 0,33 , ce qui permet de proposer, dans le cas d'un module de réaction sensiblement constant, la formule approchée :

$$
a=1,7 \cdot\left(E I \cdot \alpha / E_{M}\right)^{0.33}
$$

\section{6}

\section{Méthode d'évaluation de $\mathrm{k}$}

D'après ce qui précède, une méthode empirique d'évaluation de $\mathrm{k}$ à partir de l'essai pressiométrique pourrait se résumer à :

- évaluer a à partir de la formule (9), en tenant compte d'une borne supérieure égale aux $2 / 3$ de la fiche :

- obtenir une valeur minimale de $\mathrm{k}$ à partir des formules (2) ou (7);

- affecter la valeur minimale ainsi obtenue d'un coefficient multiplicateur égal à 3 pour tenir compte forfaitairement, faute de mieux, de la non-linéarité du comportement du sol.

Dans le cas où l'on applique (7), cette procédure se réduit à l'emploi d'une simple formule :

$$
k=2,1 \cdot\left(E_{M} / \alpha\right)^{4 / 3} / E^{1 / 3}
$$

Si l'on assimile, conformément aux recommandations de la Société internationale de mécanique des sols et travaux de fondations [1], le rapport $E_{M} / \alpha$ au module œdométrique du sol, on peut encore écrire les formules (7), (9) et (10) sous la forme :

$$
\begin{gathered}
k_{\min }=1,2 \cdot \mathrm{E}_{\text {ced }} / \mathrm{a} \\
\mathrm{a}=1,7 \cdot\left(\mathrm{EI} / \mathrm{E}_{\mathrm{\alpha ed}}\right)^{0,33} \\
\mathrm{k}=2,1 \cdot\left(\mathrm{E}_{\mathrm{cod}}\right)^{4 / 3} / \mathrm{EI}^{1 / 3}
\end{gathered}
$$

Cette formulation générale est intéressante dans la mesure où elle permet de faire le lien non seulement 
avec les essais de laboratoire, mais encore avec d'autres essais in situ, tels le pénétromètre statique, compte tenu des corrélations existant entre modules d'élasticité et résistance de pointe.

\section{Remarque}

Si l'on admet que le module d'Young du sol, $\mathrm{E}_{\mathrm{s}}$, est égal aux $2 / 3$ du module œdométrique (ce qui correspond à l'hypothèse classique d'une valeur du coefficient de Poisson égale à 1/3), on en déduit une condition supplémentaire permettant d'obtenir un minorant de $k$ dans le cas d'une fouille de faible largeur $b$; en effet la condition

$$
\mathrm{k}>2 . \mathrm{E}_{\mathrm{s}} / \mathrm{b}
$$

permet alors d'écrire, compte tenu de (11):

$$
a<0,9 . b
$$

Cette condition s'ajoute à

$$
\mathrm{a}<2 \sqrt{3} \text {. f }
$$

où f est la fíche du soutènement.

On voit ainsi que l'utilisation des formules globales (10) et (13) peut, dans certains cas, conduire à une sous-évaluation de $k$, d'où l'intérêt d'appliquer la procédure précédemment décrite, incluant l'évaluation explicite de a.

D'une façon plus générale, il ne faut pas hésiter, dans la mesure où $k$ dépend des conditions aux limites du projet, à remettre en cause les formulations globales, quelles qu'elles soient, dans tous les cas particuliers où le massif de sol n'est pas assimilable à un milieu semi infini : un autre exemple significatif en est celui des risbermes élancées, dont la souplesse n'est prise en compte par aucune formulation classique du coefficient de réaction, et qui ne peuvent donc être calculées qu'au moyen d'une méthode spécifique [9].

\section{Conclusion}

L'approche proposée, exclusivement empirique, n'a d'autre prétention que de permettre une évaluation rapide et sans risque d'erreur importante du coefficient $k$, dans tous les cas où l'usage de ce coefficient est approprié.

L'interprétation des résultats expérimentaux a été conduite de façon délibérément prudente de façon à éviter, dans l'optique d'une généralisation, tout risque de surévaluation excessive.

On peut ainsi constater, en comparant les valeurs expérimentales de k à celles résultant de la formule (10), que cette dernière sous-estime $k$ dans $50 \%$ des cas, et que l'écart relatif entre prévisions et valeurs réelles se situe dans l'intervalle $(-50 \%,+100 \%)$, c'est-à-dire qu'il est encore possible de se tromper dans un rapport de 1 à 2, mais plus dans un rapport de 1 à 10 .

Compte tenu des hypothèses très simplificatrices qui sont à la base de la méthode de calcul élasto-plastique, d'une part, et compte tenu de la faible sensibilité des valeurs des sollicitations aux variations de $k$, d'autre part, il nous semble que cette relative imprécision peut être considérée comme acceptable.

\section{Bibliographie}

(1) Amar S., Clarke B.G.F., Gambin M.P. Orr T.L.L. - Utilisation des résultats des essais pressiométriques pour le dimensionnement des fondations en Europe. $1^{\text {re }}$ partie : pressiornètre Ménard et pressiometre autoforeur. SIMSTF. Comité technique régional européen $n^{\circ} 4$, Pressiomètres, A.A. Balkema 1991

[2] Balay J. - Recommandations pour le choix des paramètres de calcul des écrans de soutènement par la méthode aux modules de réaction. LCPC, FAER 10712.1984

[3] Gigan J.-P. - Expérimentation d'un rideau de palplanches ancré par tirants actifs. Bulletin de liaison des laboratoires des Ponts et Chaussées, $n^{\circ} 129$,
1984.

[4] Gouvenot D. Bustamante M. Mesures in situ sur les ouvrages maritimes de soutènement. Annales ITBTP $n^{\circ} 375$, Série Sols et Fondations, $n^{\circ} 167$, 1979

[5) Marche R. - Sollicitation en flexion des pieux par les couches qu'ils traversent, thèse de doctorat. École Polytechnique Fédérale de Lausanne, 1974.

[6] Ménard L. Bourdon C. - Calcul des rideaux de soutènement. Méthode nouvelle prenant en compte les conditions réelles d'encastrement. Sols-Soils, $n^{\circ} 12,1965$

[7] Monnet A. - Module de réaction, coefficient de décompression, au sujet des paramètres utilisés dans la méthode de calcul élasto-plastique des soutènements. Revue Française de Géotechnique, n 66, 1994

[8] Schmitt P. - Etude expérimentale de la sollicitation exercée par le sol sur les ouvrages de soutènement souples. Revue Française de Géotechnique, n 28, 1984.

[9] Schmitt P. Bazin E., Gilbert C. Ragu J.-E. - Effet des chargements non uniformes sur les écrans de soutènement. XIII ICSMFE, New Delhi, 1994.

[10] Terzaghi K. - Evaluation of coefficients of subgrade reaction. Geotechnique, 1955

[11] Westergaard H.M. - Stresses in concrete pavements computed by theoretical analysis. Publics Roads, 1926. 